\title{
BMJ Open Impact of screen time on mental health problems progression in youth: a 1-year follow-up study
}

Xiaoyan Wu, ${ }^{1,2}$ Shuman Tao, ${ }^{1}$ Shichen Zhang, ${ }^{1,2}$ Yukun Zhang, ${ }^{1}$ Kaihua Chen, ${ }^{3}$ Yajuan Yang, ${ }^{4}$ Jiahu Hao, ${ }^{1,2}$ Fangbiao $\mathrm{Tao}^{1,2}$

To cite: Wu X, Tao $S$, Zhang $S$, et al. Impact of screen time on mental health problems progression in youth: a 1-year follow-up study. BMJ Open 2016;6: e011533. doi:10.1136/ bmjopen-2016-011533

- Prepublication history for this paper is available online. To view these files please visit the journal online (http://dx.doi.org/10.1136/ bmjopen-2016-011533).

$\mathrm{XW}$ and ST contributed equally.

Received 17 February 2016 Revised 16 September 2016 Accepted 19 October 2016

CrossMark

For numbered affiliations see end of article.

Correspondence to Dr Fangbiao Tao; fbtao@ahmu.edu.cn

\section{ABSTRACT}

Objectives: We examined the relationships between screen time (ST) and mental health problems and also increment of ST and progression of mental health problems in a college-based sample of Chinese youth. Methods: We assessed 2521 Chinese college freshmen from October 2013 to December 2014. At baseline, the mean age of participants was 18.43 years (SD 0.96 years), and 1215 (48.2\%) participants reported ST $>2 \mathrm{~h} /$ day. We estimated multivariableadjusted ORs by using logistic regression models for the risk of developing mental health problems (anxiety, depression and psychopathological symptoms) and/or progression of these problems, according to baseline ST exposure and changes in exposure at follow-up.

Results: At baseline, when ST $>2$ h/day was compared with ST $\leq 2 \mathrm{~h} /$ day, the OR was $1.38(95 \% \mathrm{Cl}$ 1.15 to 1.65$)$ for anxiety, $1.55(95 \% \mathrm{Cl} 1.25$ to 1.93$)$ for depression and $1.49(95 \% \mathrm{Cl} 1.22$ to 1.83$)$ for psychopathological symptoms. The results remained unchanged for depressive and psychopathological symptoms but not for anxiety, after additional adjustment for sex, age, residential background, body mass index, perceived family economy, sleep quality, smoking, alcohol intake, exercise after school and physical activity. When participants who had increased their ST exposure to $>2 \mathrm{~h} /$ day were compared with those with no change and ST $\leq 2 \mathrm{~h} /$ day, the OR was $1.78(95 \% \mathrm{Cl} 1.12$ to 2.83$)$ for anxiety, $1.92(95 \% \mathrm{Cl}$ 1.23 to 2.83$)$ for depression and $1.93(95 \% \mathrm{Cl} 1.16$ to $3.21)$ for psychopathological symptoms. These associations also remained after additional adjustment. Conclusions: The overall effects are consistent yet small for ST/ST increment on mental health problems and its progression. Given the small effect size of the current results, it remains unclear the degree to which ST is a practically significant risk factor for mental health outcomes. Future studies of high quality are necessary to further examine this association and the direction of causality.

\section{INTRODUCTION}

Mental health problems affect 10-20\% of young people worldwide. ${ }^{1}$ The peak age of onset of mental illness is adolescence and early adulthood. ${ }^{2}$ For many young people, the

\section{Strengths and limitations of this study}

- This study used a longitudinal design to examine the association between screen time (ST) and mental health problems in college students; few studies are available that have investigated these associations.

- It is the first study to report that participants who increased their ST exhibited a higher risk of mental health problems at follow-up.

- The current study cannot distinguish between different types of ST exposure and their effect on mental health because the questionnaires combined different types of ST in the same question.

- Given the small effect size of the current results, it remains unclear the degree to which ST is a practically significant risk factor for mental health outcomes.

college years represent a developmentally challenging transition to adulthood. Mental disorders are prevalent among college students, and the rates and severity of these disorders appear to be increasing. ${ }^{3}$ Epidemiological evidence shows that depression and anxiety are the most common psychiatric problems in college students. ${ }^{4}$ Youth who experience anxiety and depression have a significantly increased risk of negative physical and psychosocial outcomes, such as academic difficulties, poor interpersonal relationships, low selfesteem and suicide. ${ }^{56}$

In the last decade, the use of electronic media devices has dramatically increased among youth. ${ }^{78}$ Screen time (ST) exposure is highly popular and pervasive among young people. ${ }^{9}$ Evidence remains inconsistent regarding the impact of ST and media exposure on mental health issues. Although a majority of previous researches on the effects of media has been on its negative impact, ${ }^{10}{ }^{11}$ as described in detail elsewhere, ${ }^{12}$ high ST is a significant predictor of a higher probability of anxiety, depression and psychopathological symptoms among college students. However, 
some other studies have provided null associations ${ }^{13}$ and even considered potential benefits of media exposure on mental health. ${ }^{14}$ Overall, the association between ST and mental health was rather indeterminate, which added a more balanced perspective in the study area.

The present study aimed to examine the relationships between ST and mental health problems (anxiety, depression and psychopathological symptoms) in a college-based sample of Chinese youth. It was hypothesised that a longer duration of ST would be associated with more of the above-mentioned mental health problems, and increased ST exposure during follow-up might be a risk factor for the development and/or progression of mental health problems.

\section{METHODS}

\section{Participants}

This study is an extension of a cross-sectional study we reported previously. ${ }^{12}$ The present follow-up study included a study population of college freshmen (2913 at baseline) and was conducted between October 2013 and December 2014. Questionnaires were administered at the beginning and end of the study. Participants who responded to the baseline questionnaire were recruited for the follow-up; 157 participants were lost to follow-up (94.6\% retention rate), and 56 did not respond to the follow-up questionnaire. In total, 2586 questionnaires were eligible for analysis; 65 of these were invalid in terms of connection with baseline questionnaires. The final sample comprised 2521 participants with baseline and 1-year follow-up information (figure 1). The study was approved by the Ethics Committee of Anhui Medical University. Informed consent was obtained from all participants.

Figure 1 Flow chart of study participants.

\section{Assessment of ST}

Participants reported their ST in response to the question "How many hours per day do you spend on a computer (including playing video or computer games or using a computer for something that is not school work) and watching $\mathrm{TV} /$ video programs on a usual weekday and on a usual weekend day?" We calculated the average ST per week at baseline by multiplying the average reported weekday ST by 5 and average weekend day ST by 2 , and summing the two values then divided by 7. Daily ST was categorised as $\leq 2$ hours/day or $>2$ hours/day. We calculated combined scores for weekly ST at baseline and changes in ST during the follow-up period (categorised in two groups: participants who reduced or maintained constant ST exposure and those who increased ST exposure). In total, we obtained four categories of cross-stratified ST exposure for baseline ST and changes in ST. Participants with ST $\leq 2$ hours/day at baseline and who did not increase their ST exposure at follow-up were set as the reference category.

\section{Assessment of mental health problems \\ Anxiety}

Anxiety was assessed using the Self-Rating Anxiety Scale (SAS), a standard assessment instrument for which the reliability and validity have been examined in a Chinese population. ${ }^{15}$ The SAS is a 20 -item self-report assessment, each question is scored on a Likert-type scale of 1-4: never or a little of the time, some of the time, good part of the time, most of the time or always. Raw score is summed up by the score of each question, and the standardised score is calculated by int $(1.25 \times$ raw score). A total standard score of 50 was set as the cut-off point for anxiety. In this study, Cronbach's $\alpha$ coefficient was both 0.80 at baseline and follow-up. 


\section{Depression}

Depression was assessed using the Center for Epidemiologic Studies Depression Scale (CES-D). The CES-D is a commonly used, freely available self-report measure for depressive symptoms, presented in a 4-factor 20-item structure. ${ }^{16}$ All CES-D questions have four response options: rarely or none of the time $(<1$ day), some or a little of the time (1-2 days), occasionally or a moderate amount of the time (3-4 days) and most or all of the time (5-7 days). Higher CES-D scores indicate greater depressive symptoms. A total standard score of 16 was set as the cut-off point for depression. ${ }^{17}$ In this study, Cronbach's $\alpha$ coefficients were 0.87 and 0.88 at baseline and follow-up, respectively.

\section{Psychopathological symptoms}

Psychopathological symptoms were measured using the Multidimensional Sub-health Questionnaire of Adolescents (MSQA), ${ }^{18}$ a self-report screening tool that investigates symptoms experienced during the last 3 months. The MSQA consists of 39 questions on three dimensions: 17 items for emotional symptoms (eg, "Do you always feel nervous?"), 9 items for behavioural symptoms (eg, "Do you always have the impulse to damage something?") and 13 items for social adaptation problems (eg, "Were you always not suited to school life?"). All questions have six response options corresponding to the duration of each symptom (none or last $<1$ week, last $\geq 1$ weeks, last $\geq 2$ weeks, last $\geq 1$ month, last $\geq 2$ months, last $\geq 3$ months). In calculating scores, the symptom duration 'last $\geq 1$ week' was transformed into score of " 1 ' ( positive items), 'none or last $<1$ week' were transformed into score of ' 0 ' (negative items). The criterion for psychopathological symptoms was eight or more scores of ' 1 '. 19 The validity and reliability of the MSQA has been previously confirmed, ${ }^{20}$ with a Cronbach's $\alpha$ coefficient of 0.96 .

\section{Progression of mental health problems}

The progression of mental health problems were characterised as mental health problems that did not appear at baseline, but appeared during follow-up; as well as participants who have mental health problems at baseline and have increased scores of their mental health problems during follow-up.

\section{Assessment of covariates}

Age, sex, residential background, body mass index (BMI), perceived family economic status and health-related habits (including smoking, alcohol intake, sleeping behaviour, exercise after school and physical activity (PA)) were included as covariates. PA was assessed with a reliable measure used extensively in the USA as part of the 2013 Youth Risk Behaviour Survey. The question used to assess PA was "On how many of the past 7 days did you do exercises to strengthen or tone your muscles, such as push-ups, sit-ups, or lifting weights?" Response options ranged from 0 to 7 days.
High PA was defined as at least three days per week of exercise. Exercise after school was investigated by asking "Do you have the habit to do exercise after school? (response yes or no)".

Perceived economic status was also assessed by asking the question, "How do you describe your family income compared with your classmates?" Participants were asked to choose one of the three answers (low level, medium level or high level).

Smoking and alcohol intake were investigated by asking "Have you ever tried cigarette smoking, even one or two puffs? (response yes or no)" and "During your life, have you ever had at least one drink of alcohol? (response yes or no)," respectively.

Sleep problems were measured with the Pittsburgh Sleep Quality Index, a self-rated questionnaire that assesses sleep quality and disturbances over a 1-month period. Nineteen individual items generate seven component scores: subjective sleep quality, sleep latency, sleep duration, habitual sleep efficiency, sleep disturbances, use of sleeping medication and daytime dysfunction. The sum of the seven component scores yields a global score ranging from 0 to 21; higher scores indicate worse sleep quality. In a Chinese population, a score of more than 7 indicates poor sleep quality.

Height and weight were self-reported, and BMI was calculated by dividing weight in kilograms $(\mathrm{kg})$ by height in metres square $\left(\mathrm{m}^{2}\right)$.

\section{Statistical analysis}

Statistical analyses were performed with SPSS V.13.0 (SPSS for Windows, V.13.0; SPSS ., Chicago, Illinois, USA). $\chi^{2}$ tests were performed to test for differences in characteristics between ST exposure groups for categorical variables, and t-tests were used for continuous variables. We used logistic regression models to assess the relationships between mental health problems and ST at baseline, after adjusting for potential confounding variables including sex, age, residential background, BMI, perceived family economy, sleep quality, smoking, alcohol intake, exercise after school and PA. We estimated multivariable-adjusted ORs with 95\% CIs for the risk of progression of mental health problems according to baseline ST exposure and changes in exposure. The category ' $\leq 2$ hours/day' was used as the reference category for the first exposure, and 'no change' as the reference category for the second exposure.

To assess the associations for the joint exposure for baseline ST and changes in ST during follow-up, we used four categories and estimated three ORs. The lowest exposure ( $\leq 2$ hours/day and reduced/maintained mental health problems) was the reference category.

\section{RESULTS}

Participant characteristics $(\mathrm{n}=2521)$ according to ST exposure are presented in table 1 . Overall, $47.1 \%$ of the 
Table 1 Characteristics of participants according to their screen time exposure

\begin{tabular}{|c|c|c|c|c|}
\hline & \multicolumn{2}{|c|}{ Screen time (hours/day) } & \multicolumn{2}{|c|}{ Increased use } \\
\hline & $\leq 2$ hours/day & $>2$ hours/day & No & Yes \\
\hline $\mathrm{n}$ & 1306 & 1215 & 1435 & 1086 \\
\hline Female (\%) & 52.7 & 53.1 & 54.1 & 51.2 \\
\hline Urban (\%) & 44.8 & 46.2 & 45.9 & 44.9 \\
\hline Low family income (\%) & 33.5 & 28.3 & 29.1 & 33.5 \\
\hline Age (SD), years & $18.47(0.99)$ & $18.39(0.93)$ & $18.39(0.94)$ & $18.48(0.98)$ \\
\hline BMI (SD), $\mathrm{kg} / \mathrm{m}^{2}$ & $20.46(2.63)$ & 20.39 (2.65) & 20.37 (2.64) & $20.50(2.63)$ \\
\hline Poor sleep quality (\%) & 9.1 & 9.2 & 9.7 & 8.5 \\
\hline Smoking (\%) & 22.1 & 29.2 & 27.2 & 23.3 \\
\hline Alcohol intake (\%) & 55.7 & 48.2 & 63.3 & 56.4 \\
\hline Exercise after school (\%) & 59.6 & 51.1 & 52.4 & 59.6 \\
\hline High PA (\%) & 33.8 & 30.6 & 31.1 & 33.7 \\
\hline
\end{tabular}

participants were men with a mean age of 18.53 years (SD 1.00 year); $52.9 \%$ were women with a mean age of 18.34 years (SD 0.91 years). At baseline, ST exposure was more frequent among female participants and urban participants. A slightly higher prevalence of current smoking was observed in the group with ST $>2$ hours/ day. The prevalence of anxiety, depression and psychopathological symptoms at baseline were $13.0 \%, 15.5 \%$ and $18.4 \%$, respectively. The progression in anxiety was seen in $317(12.6 \%)$ participants, depression in 309 (12.3\%) participants and psychopathological symptoms in $238(9.4 \%)$ participants. The remaining participants either did not experience any increment in existing problems or did not develop new mental health problems.

As shown in table 2, at baseline, high ST was significantly positively associated with anxiety $(\mathrm{OR}=1.38 ; 95 \%$ CI 1.15 to 1.65$)$, depression ( $\mathrm{OR}=1.55 ; 95 \%$ CI 1.25 to 1.93) and psychopathological symptoms (OR=1.49; $95 \%$ CI 1.22 to 1.83$)$. The association was maintained for depression (OR=1.58; 95\% CI 1.25 to 1.99$)$ and psychopathological symptoms (OR=1.44; 95\% CI 1.17 to 1.79$)$ after adjusting for sex, age, residential background, BMI, perceived family economic status, sleep quality, smoking, alcohol intake, exercise after school and PA.

When assessing mental health problem risk according to the joint exposure to average ST exposure at baseline (two categories) and increased ST exposure during follow-up (two categories), we found that participants who increased their ST exposure exhibited higher ORs within each category of baseline use. These associations remained after additional adjustment (table 3).

\section{DISCUSSION}

In this follow-up study, we investigated ST and mental health progression in Chinese college students. We found that higher ST exposure or an increment in ST exposure at follow-up was significantly associated with a higher risk of mental health problems. These associations between ST and mental health problems progression were small yet statistically significant. To the best of our knowledge, this is the first longitudinal study to assess the association between ST and progression of mental health problems among Chinese youth.

Several environmental factors have been associated with the increasing prevalence of mental health problems among youth, including insufficient $\mathrm{PA},{ }^{21}$ unhealthy diet, ${ }^{22}$ high frequency of internet use,${ }^{23}$ long duration of sedentary time ${ }^{2425}$ and leisure-time screen use. ${ }^{26}$ Given that young people at college have higher educational levels and are likely to possess more electronic products, they may be more susceptible to ST exposure. Although our study correlated ST with mental health issues, our results were similar to the weaker associations found in previous studies. ${ }^{6}{ }^{9}$ In a Chinese high school sample, ${ }^{6}$ high ST was correlated with depressive symptoms (OR=1.52, 95\% CI 1.31 to 1.76$)$ and anxiety symptoms $(\mathrm{OR}=1.36,95 \%$ CI 1.18 to 1.57$)$. In a population-based cohort study of Danish adolescents, ${ }^{9}$ each additional hour/day spent watching television $(\mathrm{OR}=1.64,95 \%$ CI 1.18 to 2.27$)$ or screen viewing $(\mathrm{OR}=1.58,95 \%$ CI 1.18 to 2.12$)$ was associated with greater odds of prevalent depression. A Canadian study indicated that adolescents who exceeded 2 hours/day of ST had $30-50 \%$ greater odds of suboptimal self-rated mental health. ${ }^{27}$ Similarly, Trinh $e t a l^{28}$ found that high ST was associated with poorer mental health.

Nevertheless, our study is contrary to some other studies. Ferguson concluded from 101 studies that there was no relationship between video game exposure and mental health symptoms in youth. ${ }^{29}$ Similarly, the UK Millennium Cohort Study indicated that TV but not electronic games predicted a small increase in conduct problems, while ST did not predict other aspects of mental health. ${ }^{30}$ Small correlations between different levels of electronic game engagement and psychosocial adjustment were found by Przybylski among young people aged 10-15 years and noted that they are unlikely to be of practical significance. ${ }^{31}$ In the current study, the associations between ST and mental health problems progression were small yet statistically significant among Chinese college students. These existing but 
Table 2 Association between screen time and risk of mental health at base line

\begin{tabular}{|c|c|c|c|c|c|c|c|c|c|}
\hline & \multicolumn{3}{|l|}{ Anxiety } & \multicolumn{3}{|c|}{ Depression } & \multicolumn{3}{|c|}{ Psychopathological symptoms } \\
\hline & n (\%) & $\begin{array}{l}\text { Crude OR } \\
(95 \% \mathrm{Cl})\end{array}$ & $\begin{array}{l}\text { Adjusted OR } \\
(95 \% \mathrm{Cl})\end{array}$ & n (\%) & $\begin{array}{l}\text { Crude OR } \\
(95 \% \mathrm{Cl})\end{array}$ & $\begin{array}{l}\text { Adjusted OR } \\
(95 \% \mathrm{Cl})\end{array}$ & n (\%) & $\begin{array}{l}\text { Crude OR } \\
(95 \% \mathrm{Cl})\end{array}$ & $\begin{array}{l}\text { Adjusted OR } \\
(95 \% \mathrm{Cl})\end{array}$ \\
\hline \multicolumn{10}{|l|}{ Screen time } \\
\hline$\leq 2$ hours/day & $156(11.9)$ & Ref. & Ref. & $225(18.5)$ & Ref. & Ref. & $203(15.5)$ & Ref. & Ref. \\
\hline$>2$ hours/day & $171(14.1)$ & $\begin{array}{l}1.38 \\
(1.15 \text { to } 1.65) \dagger\end{array}$ & $\begin{array}{l}1.25 \\
(0.98 \text { to } 1.60)\end{array}$ & $167(12.8)$ & $\begin{array}{l}1.55 \\
(1.25 \text { to } 1.93) \dagger\end{array}$ & $\begin{array}{l}1.58 \\
(1.25 \text { to } 1.99) \dagger\end{array}$ & $262(21.6)$ & $\begin{array}{l}1.49 \\
(1.22 \text { to } 1.83) \dagger\end{array}$ & $\begin{array}{l}1.44 \\
(1.17 \text { to } 1.79) \dagger\end{array}$ \\
\hline
\end{tabular}

${ }^{*}$ Adjusted for sex, age, residential background, BMI, perceived family economy, sleep quality, smoking, alcohol intake, exercise after school and PA.

tp<0.05 compared with referent.

BMI, body mass index; PA, physical activity.

Table 3 Association between a combined score of screen time and risk of mental health increment

\begin{tabular}{|c|c|c|c|c|c|c|c|}
\hline \multirow[b]{2}{*}{ Increment of mental health } & \multirow{2}{*}{$\begin{array}{l}\text { Increased use } \\
\text { during follow-up }\end{array}$} & \multicolumn{3}{|c|}{ Screen time $\leq 2$ hours/day } & \multicolumn{3}{|c|}{ Screen time $>2$ hours/day } \\
\hline & & Percent & Crude OR (95\% Cl) & Adjusted OR $(95 \% \mathrm{Cl})^{*}$ & Percent & Crude OR $(95 \% \mathrm{Cl})$ & Adjusted OR (95\% Cl) \\
\hline \multirow[t]{2}{*}{ Anxiety } & No & 9.6 & Ref. & Ref. & 11.1 & $1.18(0.81$ to 1.70$)$ & $1.20(0.84$ to 1.73$)$ \\
\hline & Yes & 12.4 & $1.34(0.92$ to 1.94$)$ & $1.34(0.94$ to 1.94$)$ & 15.8 & $1.78(1.12$ to 2.83$) \dagger$ & 1.77 (1.12 to 2.79$) \dagger$ \\
\hline \multirow[t]{2}{*}{ Depression } & No & 10.2 & Ref. & Ref. & 10.1 & $0.98(0.68$ to 1.42$)$ & $1.02(0.72$ to 1.46$)$ \\
\hline & Yes & 11.2 & $1.11(0.77$ to 1.61$)$ & $1.13(0.79$ to 1.63$)$ & 17.9 & $1.92(1.23$ to 3.00$) \dagger$ & 1.98 (1.28 to 3.05$) \dagger$ \\
\hline \multirow[t]{2}{*}{ Psychopathological symptoms } & No & 7.4 & Ref. & Ref. & 7.4 & $1.00(0.65$ to 1.53$)$ & $1.00(0.67$ to 1.49$)$ \\
\hline & Yes & 8.9 & $1.22(0.80$ to 1.86$)$ & $1.16(0.78$ to 1.74$)$ & 13.3 & $1.93(1.16$ to 3.21$) \dagger$ & 1.76 (1.07 to 2.87$) \dagger$ \\
\hline
\end{tabular}


small links are controversial, accompanied by increasingly careful conclusions made to avoid broad statements that directly link ST exposure to mental health problems. In a similar vein, the overall effects are consistent yet small for ST/ST incensement on mental health problems and its progression, indicating that the broad fears about ST may be exaggerated. ${ }^{31}$ It is also notable that no causal inferences can be made, just as likely that youth who are experiencing mental health symptoms may turn to TV and video games to soothe themselves. However, our study suggests that a cautionary approach to the high exposure of ST in young people is justifiable in terms of potential effects on mental health.

There is little research evidence available as yet on the relationships between increment in ST and progression of mental health problems in youth. The major strength of this study was the use of data for the same sample from two time points, at which ST and mental health changes were investigated. In addition, important confounders were included in analysis, helping to clarify the association between ST and mental health in a follow-up population.

Several limitations of the present study should be recognised. First, ST exposure and outcome variables relied on self-reported data. However, our follow-up sample was highly educated and cooperative college students. Therefore, we believe that the quality and validity of the self-reported data provided by participants were reliable. Second, the short follow-up period might have influenced the results. Future long-term prospective studies are required to delineate the effects of ST on mental health. Third, there are some potential limitations to this study and results should be cautiously interpreted. The authors acknowledged that there is potential for type I error with small effect sizes; therefore, it remains unclear the degree to which ST is a practically significant risk factor for mental health outcomes. Finally, we could not distinguish between different types of ST exposures and their effect on mental health because the questionnaires combined different types of ST in the same question. Little is known on how specific ST activities relate to mental health in youth, although such relationships are important, given that specific types of ST play different roles in mental health. ${ }^{32} 33$ Previous studies have indicated that time spent on computer and video games may be more common than time spent on watching $\mathrm{TV} .{ }^{10}$ Future studies are needed that investigate different types of ST and the effects on mental health associated with each type of ST.

\section{CONCLUSIONS}

The associations between ST and mental health problems progression were small yet statistically significant among Chinese college students. Participants who had increased ST at follow-up exhibited a higher risk of mental health problems. The overall effects are consistent yet small for ST/ST incensement on mental health problems and its progression. Given the small effect size of the current results, it remains unclear the degree to which ST is a practically significant risk factor for mental health outcomes. Future studies of high quality are necessary to further examine these associations and the direction of causality.

\section{Author affiliations}

${ }^{1}$ Department of Maternal, Child and Adolescent Health, School of Public Health, Anhui Medical University, Hefei, China

${ }^{2}$ Anhui Provincial Key Laboratory of Population Health and Aristogenics, Hefei, China

${ }^{3}$ Department of Physical Education, School of General Education, Anhui Medical University, Hefei, China

${ }^{4}$ Department of Surgical Nursing, School of Nursing, Anhui Medical University, Hefei, China

Acknowledgements This study was a joint effort of school teachers. The authors thank them for the help in conducting this study. They specially thank the students who participated in the study. The authors are thankful to Edanz Editing Company for the help of improving the manuscript.

Contributors FT contributed to study design; ST and SZ analysed the data; YZ and $\mathrm{KC}$ contributed reagents/materials/analysis tools; $\mathrm{YY}$ and $\mathrm{JH}$ performed the experiments and XW wrote the paper and contributed to study design. All authors interpreted the data and revised the manuscript critically for important intellectual content. They also read and approved the final version of the manuscript.

Funding The authors are grateful for the financial support offered by the National Natural Science Foundation of China (grant number 81302448) and Anhui Provincial Natural Science Foundation (grant number 1408085MKL30).

Competing interests None declared.

Provenance and peer review Not commissioned; externally peer reviewed.

Data sharing statement No additional data are available.

Open Access This is an Open Access article distributed in accordance with the Creative Commons Attribution Non Commercial (CC BY-NC 4.0) license, which permits others to distribute, remix, adapt, build upon this work noncommercially, and license their derivative works on different terms, provided the original work is properly cited and the use is non-commercial. See: http:// creativecommons.org/licenses/by-nc/4.0/

\section{REFERENCES}

1. Jurewicz I. Mental health in young adults and adolescentssupporting general physicians to provide holistic care. Clin Med 2015;15:151-4.

2. Kessler RC, Berglund $\mathrm{P}$, Demler $\mathrm{O}$, et al. Lifetime prevalence and age-of-onset distributions of DSM-IV disorders in the National Comorbidity Survey Replication. Arch Gen Psychiatry 2005;62:593-602

3. Hunt J, Eisenberg D. Mental health problems and help-seeking behavior among college students. J Adolesc Health 2010;46:3-10.

4. Kirsch DJ, Doerfler LA, Truong D. Mental health issues among college students: who gets referred for psychopharmacology evaluation? J Am Coll Health 2015;63:50-6.

5. Hawgood J, De Leo D. Anxiety disorders and suicidal behaviour: an update. Curr Opin Psychiatry 2008;21:51-64.

6. Cao H, Qian Q, Weng T, et al. Screen time, physical activity and mental health among urban adolescents in China. Prev Med 2011;53:316-20.

7. Minges KE, Owen N, Salmon J, et al. Reducing youth screen time: qualitative metasynthesis of findings on barriers and facilitators. Health Psychol 2015;34:381-97.

8. Cui Z, Hardy LL, Dibley MJ, et al. Temporal trends and recent correlates in sedentary behaviours in Chinese children. Int $J$ Behav Nutr Phys Act 2011;8:93.

9. Grøntved A, Singhammer J, Froberg K, et al. A prospective study of screen time in adolescence and depression symptoms in young adulthood. Prev Med 2015;81:108-13. 
10. Maras D, Flament MF, Murray M, et al. Screen time is associated with depression and anxiety in Canadian youth. Prev Med 2015;73:133-8.

11. Feng $Q$, Zhang QL, Du Y, et al. Associations of physical activity, screen time with depression, anxiety and sleep quality among Chinese college freshmen. PLoS One 2014;9:e100914.

12. Wu XY, Tao SM, Zhang YK, et al. Low physical activity and high screen time can increase the risks of mental health problems and poor sleep quality among Chinese college students. PLoS One 2015;10:e0119607.

13. Merritt A, LaQuea L, Cromwell R, et al. Media managing mood: a look at the possible effects of violent media on affect. Child Youth Care Forum 2016;45:241-58.

14. Granic I, Lobel A, Engels RC. The benefits of playing video games. Am Psychol 2014;69:66-78.

15. Zung WW. A rating instrument for anxiety disorders. Psychosomatics 1971;12:371-9.

16. Ling Y, Wei Y, Yi JY, et al. Factorial structure of the CES-D Scale among Chinese high school students. Chin J Clin Psychol 2008;16:265-7.

17. Radloff LS. The use of the Center for Epidemiologic Studies Depression Scale in adolescents and young adults. $J$ Youth Adolesc 1991;20:149-66.

18. Tao FB, Hu CL, Sun YH, et al. The development and application of multidimensional sub-health questionnaire of adolescents (MSQA). Chin J Dis Control Prev 2008;12:309-14.

19. Liu XC, Tang MQ, Hu L, et al. Reliability and validity of Pittsburgh Sleep Quality Index. Chin J Psychiatry 1996;29:103-7.

20. Xing C, Tao FB, Yuan CJ, et al. Evaluation of reliability and validity of the multidimensional sub-health questionnaire of adolescents. Chin J Public Health 2008:24:1031-3.

21. Kim YS, Park YS, Allegrante JP, et al. Relationship between physical activity and general mental health. Prev Med 2012:55:458-63.

22. Daley C, Patterson A, Sibbritt D, et al. Unsaturated fat intakes and mental health outcomes in young women from the Australian Longitudinal Study on Women's Health. Public Health Nutr 2015;18:546-53.
23. Kalckreuth S, Trefflich F, Rummel-Kluge C. Mental health related internet use among psychiatric patients: a cross-sectional analysis. BMC Psychiatry 2014;14:368

24. Hamer M, Coombs N, Stamatakis E. Associations between objectively assessed and self-reported sedentary time with mental health in adults: an analysis of data from the Health Survey for England. BMJ Open 2014;4:e004580.

25. Teychenne M, Costigan SA, Parker K. The association between sedentary behaviour and risk of anxiety: a systematic review. BMC Public Health 2015;15:513.

26. Kremer P, Elshaug C, Leslie E, et al. Physical activity, leisure-time screen use and depression among children and young adolescents. J Sci Med Sport 2014;17:183-7.

27. Herman KM, Hopman WM, Sabiston CM. Physical activity, screen time and self-rated health and mental health in Canadian adolescents. Prev Med 2015;73:112-16.

28. Trinh L, Wong B, Faulkner GE. The Independent and Interactive Associations of Screen Time and Physical Activity on Mental Health, School Connectedness and Academic Achievement among a population-based sample of youth. J Can Acad Child Adolesc Psychiatry 2015;24:17-24.

29. Ferguson CJ. Do angry birds make for angry children? A meta-analysis of video game influences on children's and adolescents' aggression, mental health, prosocial behavior, and academic performance. Perspect Psychol Sci 2015;10:646-66.

30. Parkes A, Sweeting $H$, Wight $D$, et al. Do television and electronic games predict children's psychosocial adjustment? Longitudinal research using the UK Millennium Cohort Study. Arch Dis Child 2013:98:341-8.

31. Przybylski AK. Electronic gaming and psychosocial adjustment. Pediatrics. 2014;134:e716-22.

32. Primack BA, Swanier B, Georgiopoulos AM, et al. Association between media use in adolescence and depression in young adulthood: a longitudinal study. Arch Gen Psychiatry 2009;66:181-8.

33. Ahmadi J, Amiri A, Ghanizadeh A, et al. Prevalence of addiction to the internet, computer games, DVD, and video and its relationship to anxiety and depression in a sample of Iranian High School Students. Iran J Psychiatry Behav Sci 2014;8:75-80. 